\title{
THORACOSCOPIC MAJOR LUNG RESECTION FOR PRIMARY LUNG CANCER BY A SINGLE SURGEON WITH A VOICE-CONTROLLED ROBOT AND AN INSTRUMENT RETRACTION SYSTEM
}

Shinichiro Okada, MD, Yoshiaki Tanaba, MD, Hiromitsu Sugawara, MD, Hideyuki Yamauchi, MD, Shotaro Ishimori, MD, and Shoichi Satoh, MD, Kamaishi, Japan

Recent advances and improvements in operative techniques, devices, and equipment have expanded the endoscopic application and allowed endoscopic performance of complex surgical procedures. Additionally, more recently, robots have been introduced into surgical procedures in an attempt to facilitate surgical performance and reduce personnel resource use. ${ }^{1,2} \mathrm{We}^{3}$ have used a robot to manipulate a thoracoscope to perform single-surgeon thoracoscopic surgery since 1997.

In this report, we describe our first experience with singlesurgeon thoracoscopic major lung resection for primary lung cancer with a voice-controlled robot used to manipulate an endoscope and an instrument retraction system.

Clinical summary. A 72-year-old woman was referred to our department for further examination of an abnormal shadow on a chest x-ray film. A computed tomogram of the chest demonstrated a $2 \times 2-\mathrm{cm}$ spiculated mass in the right lung field (Fig 1). Bronchoscopic brush cytology revealed adenocarcinoma of the lung. The clinical stage was cT1 N0 M0. After obtaining written informed consent from the patient and her family, we performed a thoracoscopic operation.

The patient was placed in the left lateral decubitus position with general anesthesia and single-lung ventilation. The robotic arm (AESOP2000; Computer Motion, Goleta, Calif) was established on the operating table posterior to the patient's thigh and the instrument retraction system (UNITRAC; Aesculap, Tuttlingen, Germany) posterior to the patient's head (Fig 2). Motion of the robotic arm was controlled by the operating surgeon with 23 distinct voice commands. The retraction and holding system for an endoscopic instrument, which was directly connected to the compressed air supply, was pneumatically supported and operated by pressing one button. The operation began with the surgeon behind the patient. When necessary, the surgeon changed position. An assistant surgeon was on hand if needed.

The first trocar was placed in the right sixth intercostal

From the Department of Thoracic Surgery and Medicine, Kamaishi Municipal Hospital, Kamaishi, Japan.

Received for publication Feb 24, 2000; accepted for publication March 6, 2000.

Address for reprints: Shinichiro Okada, MD, Department of Thoracic Surgery and Medicine, Kamaishi Municipal Hospital, Kamaishi, Iwate 026-0025, Japan

(E-mail: shin575@opal.famille.ne.jp).

J Thorac Cardiovasc Surg 2000;120:414-5

Copyright (c) 2000 by The American Association for Thoracic Surgery

$0022-5223 / 2000 \$ 12.00+0 \quad \mathbf{1 2 / 5 4 / 1 0 7 2 0 5}$

doi:10.1067/mtc.2000.107205

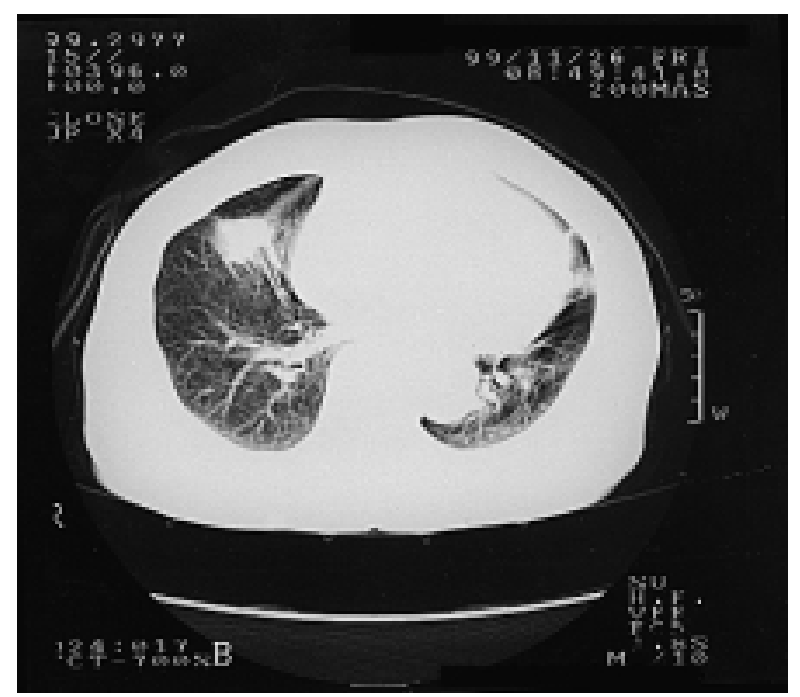

Fig 1. A computed tomogram of the chest on admission demonstrating a spiculated mass in the middle lobe of the right lung.

space at the midaxillary line. The thoracoscope connected with the robotic arm was inserted through the trocar. Under thoracosopic vision, one additional trocar was inserted and a $3-\mathrm{cm}$ incision was made in the right fourth intercostal space at the anterior axillary line and $2 \mathrm{~cm}$ posterior to the lower angle of the right scapular bone, respectively. The instruments, such as a grasper, a dissector, and an automatic stapler, which were connected to the instrument retraction system by hand control, were inserted through the posterior trocar and the small wound. Incomplete segments of the major fissure were separated with an automatic stapler. The upper lobe of the right lung was freed from adhesions with the ultrasonically activated device (Harmonic Scalpel; Johnson and Johnson, Inc, Tokyo, Japan). Through the 3-cm wound and the 2 thoracoports, the middle lobectomy was performed with hilar and mediastinal lymph node dissection. The ribs were not spread or traumatized. The pulmonary vein and middle lobe bronchus were transected with staplers and the pulmonary arteries were transected with the stapler and suture ligation through the small wound. The resected lobe was removed in a plastic bag through the $3-\mathrm{cm}$ incision in the intercostal space without a rib spreader. A 20F chest drainage tube was inserted. The solo operation was per- 
formed without the need for a human assistant. No technical operative mishaps were associated with the maneuvers of the robotic arm and the instrument retraction system. The operating time and the amount of bleeding were 317 minutes and $203 \mathrm{~g}$, respectively. The patient was extubated in the operating room. The postoperative course was satisfactory, necessitating no analgesics. The chest tube was removed on the second postoperative day. The patient returned to preoperative levels of physical activity on the seventh postoperative day. However, on her request, she was discharged to her home in good condition on the fourteenth postoperative day.

Discussion. Although the absolute indications for thoracoscopic surgery for patients with primary lung cancer have not yet been clearly established, several reports have described the validity of the thoracoscopic procedure for the early stages of primary lung cancer. ${ }^{4,5}$

To date, we have used the voice-controlled robot to manipulate a thoracoscope to perform single-surgeon thoracoscopic surgery in 70 cases since 1997 and found that thorascopic solo operations such as partial resection of the lung, removal of mediastinal tumors, and resection of chest wall tumors are feasible and potentially cost-effective over the long term. In the present case, we used a combination of the voice-controlled robot and the instrument retraction system and performed a purely thoracoscopic major lung resection with lymph node dissection without the need for a human assistant. No complications occurred related to the maneuvers of the robotic arm and the instrument retraction system.

In the present case, the operation took longer because of adhesions and incomplete segments of the major fissure. Additionally, the fact that this case was our first attempt with this technique may have prolonged the operation. As experience is gained, operative time should become shorter.

To our knowledge, this is the first report of thoracoscopic major lung resection by a single surgeon using robotic assistance for primary lung cancer. Although further studies on many robotically assisted procedures are necessary to clarify the clinical feasibility and utility of our procedure, the results in the present case are encouraging. We believe that thoracoscopic complicated procedures such as major lung resection with lymph node dissection using a voice-controlled robot and an instrument retraction system may be technically feasible in selected cases and in the hands of an experienced thoracic surgeon.

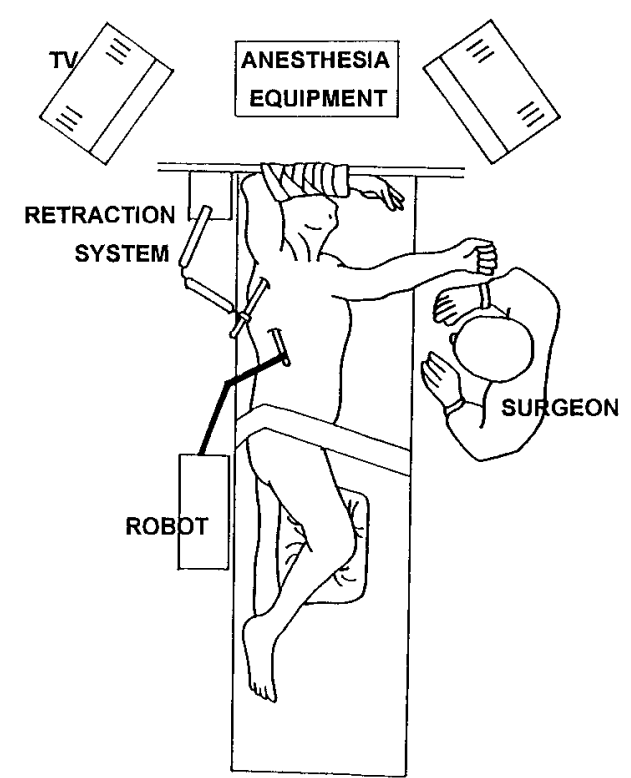

Fig 2. Position of the robot and the instrument retraction system during the thoracoscopic major lung resection. The body of the voice-controlled robot holding the thoracoscope is fixed at the operating table posterior to the patient's thigh. The instrument retraction system is mounted to the operating table behind the head of the patient.

\section{REFERENCES}

1. Partin AW, Adams JB, Moore RG, Kavoussi LR. Complete robotassisted laparoscopic urologic surgery: a preliminary report. J Am Coll Surg 1995;181:552-7.

2. Shennib H, Bastawisy A, McLoughlin J, Moll F. Robotic computer-assisted telemanipulation enhances coronary artery bypass. J Thorac Cardiovasc Surg 1999;117:310-3.

3. Okada S, Tanaba Y, Yamauchi H, Sato S. Single-surgeon thoracoscopic surgery with a voice-controlled robot. Lancet 1998; $351: 1249$.

4. Kaseda S, Hangai N, Yamamoto S, Kitano M. Lobectomy with extended lymph node dissection by video-assisted thoracic surgery for lung cancer. Surg Endosc 1997;11:703-6.

5. Sugi K, Kaneda Y, Esato K. Video-assisted thoracoscopic lobectomy achieves a satisfactory long-term prognosis in patients with clinical stage IA lung cancer. World J Surg 2000;24:27-30. 